\title{
IMPORTÂNCIA DA ASSISTÊNCIA DE ENFERMAGEM AO PACIENTE PORTADOR DE DIABETES MELLITUS TIPO DOIS
}

Freitas, William Ribeiro ${ }^{1}$

Freitas, William Ribeiro. Importância da assistência de enfermagem ao paciente portador de diabetes mellitus tipo dois. Revista Científica Multidisciplinar Núcleo do Conhecimento. Ano 03, Ed. 08, Vol. 09, pp. 32-54, Agosto de 2018. ISSN:24480959

\section{RESUMO}

Devido a diabetes mellitus ser um grande problema enfrentado pela população mundial, podendo ocasionar graves complicações se não for precocemente diagnosticada, iniciado o tratamento, se paciente não receber orientações gerais sobre o quadro, pode trazer grandes agravos a sua saúde, desse modo adaptado esse problema para a realidade da enfermagem, o trabalho solucionará os objetivos propostos, tendo como geral, relatar a importância que assistência de enfermagem possui no diagnóstico, tratamento e acompanhamento do portador de diabetes mellitus tipo dois. Este trabalho é uma revisão bibliográfica que utilizou várias fontes bibliográficas para solucionar o problema estudado e esclarecer seu objetivo geral e específicos. Pode-se concluir através deste trabalho que assistência de enfermagem e de extrema importância para a prevenção, orientação auxilio no tratamento da diabetes mellitus tipo dois em conjunto com a equipe multiprofissional.

Palavras-chave: Enfermagem; Assistência, Diabetes, Mellitus, Complicações.

\section{INTRODUÇÃO}

Diabetes mellitus é doença metabólica que causa um aumento do nível de glicose presente no sangue, ela tem registros muito antigos, seus primeiros registros são de

\footnotetext{
${ }^{1}$ Acadêmico de Enfermagem.
} 
meados do século $X V$, e com o passar do tempo vem se propagando entre a população mundial. Segundo a Federação Internacional de Diabetes (2014), 415 milhões de adultos são portadores de diabetes e em 2040 serão 642 milhões. Há vários tipos de diabetes mellitus, a mais comum, que ocorre em cerca de 90 a 95\% dos casos registrados é a diabetes mellitus tipo 2, que o tipo em enfoque desse trabalho. A diabetes tipo 2 ocorre por causa de um defeito na secreção de insulina e na sua regulação.

\section{OBJETIVOS}

Este trabalho teve como objetivo geral: relatar a importância que assistência de enfermagem possui no diagnóstico, tratamento e acompanhamento do portador de diabetes mellitus tipo dois. Irá apresentar em seus objetivos específicos pontos chaves sobre diabetes mellitus tipo 2, como seus principais aspectos: Explanar sobre a definição de diabetes mellitus tipo 2 e seus aspectos (definição, epidemiologia, fisiopatologia, prevenção, diagnóstico e tratamento); abordará as complicações crônicas que a diabetes mellitus tipo 2 pode causar: retinopatia diabética, neuropatia diabética, doença renal no diabético e irá descrever a assistência de enfermagem prestado ao diabético, desde a abordagem inicial ao paciente recém diagnosticado a condutas em complicações apresentadas.

\section{JUSTIFICATIVA}

Este trabalho teve como objetivo geral: relatar a importância que assistência de enfermagem possui no diagnóstico, tratamento e acompanhamento do portador de diabetes mellitus tipo dois. Irá apresentar em seus objetivos específicos pontos chaves sobre diabetes mellitus tipo 2, como seus principais aspectos: Explanar sobre a definição de diabetes mellitus tipo 2 e seus aspectos (definição, epidemiologia, fisiopatologia, prevenção, diagnóstico e tratamento); abordará as complicações crônicas que a diabetes mellitus tipo 2 pode causar: retinopatia diabética, neuropatia diabética, doença renal no diabético e irá descrever a assistência de enfermagem prestado ao diabético, desde a abordagem inicial ao paciente recém diagnosticado a condutas em complicações apresentadas. 


\section{METODOLOGIA}

Este é um estudo de revisão bibliográfica, que irá descreveu a assistência de enfermagem ao portador de diabetes mellitus tipo dois. Serão utilizadas como palavras-chave/frases-chave para pesquisa: assistência de enfermagem, assistência de enfermagem ao paciente diabético, diabetes mellitus, diabetes mellitus tipo dois, complicações geradas pela diabetes mellitus. Critérios de inclusão: escrito em português, inglês ou espanhol. Publicação entre 2005 e 2017, nas quais seja abordado à diabetes mellitus tipo dois. Critérios de exclusão: escrita em outras línguas diferentes das do critério de inclusão, data de publicação anterior ao ano de 2005, aborde somente diabetes tipo um. Foram selecionadas 25 fontes entre artigos, livros e diretrizes. Bases de dados/artigos utilizadas para desenvolvimento do presente trabalho: Scielo, Diretrizes da Sociedade Brasileira de Diabetes, Diretriz da Sociedade Americana de Diabetes, Veredas-Favip, Revista USP, BVS (Biblioteca Virtual em Saúde), Livro - Black Book, Livro - Harrison de Medicina Interna, Livro - Cecil de Medicina Interna, Livro - Clínica Médica (USP).

\section{ENTENDENDO A DIABETES MELLITUS TIPO 2 (DM2)}

DM (Diabetes mellitus) é um grupo de distúrbios metabólicos que tem como principal apresentação a hiperglicemia, que podem ser resultado de defeitos na ação da insulina ou na secreção da mesma. A DM pode ser classificada como tipo 1, tipo 2 , gestacional. (SBD, 2016)

O aparecimento, desenvolvimento da DM2 (diabetes mellitus tipo 2) está relacionado a resistência à insulina e a secreção anormal de insulina. A DM2 tem grande envolvimento genético para o seu desenvolvimento além do modo de vida sedentário e obesidade, pessoas com o pais portadores de DM2 tem até $40 \%$ mais chances de desenvolver a DM2. (POWERS, 2013)

Diabetes mellitus tipo 2 (tipo adulto): É a forma mais frequente (90-95\% dos casos). É uma doença crônica relacionada à resistência e perda progressiva de capacidade de secreção de insulina e, após anos sem controle adequado, causa uma série de 
complicações cardiovasculares, cerebrovasculares, renais, oftalmológicas, etc. com grande impacto de saúde pública. A doença tem forte relação com excesso de peso (sobretudo com obesidade abdominal), sedentarismo, alimentação inadequada e idade acima de 50 anos. Cerca de $6 \%$ dos brasileiros adultos têm diagnóstico de diabetes tipo 2 e essa prevalência aumenta com a idade, atingindo $22 \%$ na população com mais de 65 anos. Pelo menos um quarto dos diabéticos não sabe de sua doença e muitos morrem por complicações da doença antes do diagnóstico. (OLIVEIRA, 2014, p. 566).

O portador de DM2 tem uma perda progressiva da capacidade de secretar insulina, com a progressão da DM2 a hiperglicemia se torna mais grave e com o passar do tempo os níveis de insulina presente no sangue não conseguirão manter a glicemia em controle. (SHERWIN; INZUCCHÍ, 2011).

\subsection{EPIDEMIOLOGIA}

Diabetes mellitus é uma das doenças que tem maior crescimento na população mundial, a projeção mundial de diabetes mellitus em 2003 era de 194 milhões, e em 2025 será de 333 milhões. (SILVA et al., 2009)

O número de diabéticos está aumentando em virtude do crescimento e do envelhecimento populacional, da maior urbanização, da progressiva prevalência de obesidade e sedentarismo, bem como da maior sobrevida de pacientes com DM. Quantificar o predomínio atual de DM e estimar o número de pessoas com diabetes no futuro é importante, pois possibilita planejar e alocar recursos de maneira racional. (SBD, 2016, p. 3)

Devido a DM2 ser uma doença "silenciosa", as vezes apresentando-se de maneira assintomática, seu diagnóstico muitas vezes e feito quando o paciente já está com a doença instalada há algum tempo. O retardamento do diagnóstico as vezes se dá ao baixo grau de escolaridade, idade avançada, que interferem no processo de aprendizagem sobre a doença (PACE et al., 2006) 
O diabetes mellitus é um problema de saúde pública, que vem se alastrando cada vez mais pela população, tanto de países desenvolvidos com subdesenvolvidos. Esse aumento significativo de pessoas com diabetes, se dá pelos hábitos alimentares precários e o aumento do peso corporal. (FERREIRA; PITITTO, 2014).

Em 2012, o diabetes causou 1,5 milhão de óbitos. Um nível de glicose no sangue superior ao desejável causou outros 2,2 milhões de mortes, aumentando os riscos de enfermidades cardiovasculares e de outros tipos. (OMS, 2016, tradução nossa)

Muitas vezes na declaração de óbito, não consta DM como motivo da morte, pelo fato da mesma ter sido causada por suas complicações, entre as principais complicações cardiovasculares e cerebrovasculares. (SBD, 2016)

\subsection{FISIOPATOLOGIA}

A DM2 pode ocorre devido a ação de dois distúrbios, a diminuição da secreção de insulina pelas células beta do pâncreas e a resistência à insulina. Na DM2 ocorre um desequilíbrio dos sistemas que fazem o controle dos níveis de glicose, esse desequilíbrio inclui, aumento da produção hepática, redução da captação periférica e armazenagem de glicose, o que acarreta no aumento do das glicemias em jejum e pós-prandiais. (SILVA et al., 2009)

Devido à resistência à insulina a lipólise do tecido adiposo também aumenta, liberando na circulação ácidos graxos livres, que agravam a resistência à insulina. Por causa da grande demanda de insulina, gradativamente as células betas do pâncreas então em falência, manifestando-se em intolerância à glicose, que evoluirá para DM2. (SILVA et al., 2009)

Defeitos genéticos e metabólicos distintos na ação e/ou secreção da insulina dão origem ao fenótipo comum da hiperglicemia no DM tipo 2 e comportam importantes implicações terapêuticas potenciais agora que estão disponíveis agentes farmacológicos cujos alvos são desarranjos metabólicos específicos. O dm tipo 2 é precedido por um período de homeostasia anormal da glicose classificado como 
glicose em jejum alterada (GJA) ou tolerância diminuída à glicose (TGD). (POWERS, 2013, p. 2968)

A DM tem um grande envolvimento genético e ambiental para o seu desenvolvimento. "A hiperglicemia no diabetes tipo 2 provavelmente resulta de interações genéticas complexas, cuja expressão é modificada por fatores ambientais, como peso corporal e exercício. [...]" (SHERWIN; INZUCCHÍ, 2011, p. 2014)

Atualmente é aparente que a força impulsionadora na progressão da tolerância à glicose normal para a IG, manifestando-se em diabetes tipo 2, é a perda progressiva da capacidade secretória de insulina. Depois que o diabetes tipo 2 se manifesta, os níveis de insulina $\mathrm{cm}$ jejum geralmente parecem normais ou elevados, mesmo sendo relativamente baixos com razão do grau de hiperglicemia coexistente. (SHERWIN, 2011, p. 2015)

A tolerância à glicose nos estágios iniciais da DM2 continua quase normal, por que as células betas pancreáticas fazem uma compensação com o aumento da produção de insulina. (POWERS, 2013)

\subsection{DIAGNÓSTICO DE DM}

Assim como é mostrado no quadro 1, os valores de referência de cada aferição, mostrando glicemia normal, quando há tolerância a glicose diminuída e a DM instalada já. Esses são os valores de referência para os níveis de glicose utilizado para diagnóstico de DM. (SBD, 2016)

O diagnóstico de DM2 se dá ao aparecimento de alguns sinais e sintomas: poliúria, polidipsia e perda ponderal de peso, acompanhados de alterações do nível de glicose. Sendo levado em conta a glicemia em jejum, após teste de tolerância a glicose e casual. (NAM HAN CHO, 2015, p. 23).

E comum o relato de sede excessiva em pacientes portador de DM2. Por ser uma doença "silenciosa" muitas vezes paciente só é diagnosticado, quando está com a 
doença instalada a algum tempo, levando em conta o tempo que a hiperglicemia demora para causar danos consideráveis ao organismo

Quadro 1 - Diagnóstico de DM

\begin{tabular}{l|l|l|l|}
\hline Categoria & Jejum* & $\begin{array}{l}2 \mathrm{~h} \text { após } 75 \mathrm{~g} \\
\text { de glicose }\end{array}$ & Casual** $^{* *}$ \\
\hline $\begin{array}{l}\text { Glicemia } \\
\text { normal }\end{array}$ & $<100$ & $<140$ & \\
$\begin{array}{l}\text { Tolerância à } \\
\text { glicose } \\
\text { diminuída }\end{array}$ & $\geq 100 \mathrm{a}<126$ & $\geq 140 \mathrm{a}<200$ & \\
$\begin{array}{l}\text { Diabetes } \\
\text { mellitus }\end{array}$ & $\geq 126$ & $\geq 200$ & $\begin{array}{l}\geq 200 \text { (com }^{\text {sintomas }} \\
\text { clássicos) }\end{array}$ \\
\hline
\end{tabular}

Fonte: Diretrizes da Sociedade Brasileira de Diabetes - SBD, 2016, p. 11

É também realizado o teste de tolerância a glicose, sendo administrado $75 \mathrm{~g}$ glicose ao paciente, sendo o teste positivo se o valor da glicose após $2 \mathrm{~h} 00$ do teste ser igual ou maior a 200mg/dl. (SBD, 2016)

Uma medida de glicose plasmática em jejum de 8 horas (na noite anterior) é mais conveniente; o diabetes está estabelecido se os níveis plasmáticos de glicose em jejum forem de $126 \mathrm{mg} / \mathrm{dl}$ ou acima em duas ocasiões diferentes. Alternativamente, pode ser empregado um teste de tolerância à glicose oral de $75 \mathrm{~g}$. O teste de tolerância à glicose oral deve ser realizado após uma noite em jejum, com uso de carga de glicose contendo $75 \mathrm{~g}$ de glicose anidra dissolvida em água; os níveis de glicose póscarga em 2 horas, de $200 \mathrm{mg} / \mathrm{dl}$, ou cima, confirmam a presença de diabetes. (SHERWIN; INZUCCHÍ, 2011, p. 2016)

Outro exame utilizado para auxiliar no diagnóstico de DM é a hemoglobina glicada (HbA1c) devido a possibilidade de avaliar o grau de exposição à glicemia durante o tempo e os valores se mantém estáveis após a coleta. Tendo como valor de referência: $6,5 \%$ que deve ser confirmada com outra coleta (dispensável se paciente apresentar sintomas clássico ou glicemia igual ou maior que $200 \mathrm{mg} / \mathrm{dl}$ ). Paciente 
apresentando $\mathrm{HbA} 1 \mathrm{c}$ entre 5,7 e 6,4\% tem um risco alto de desenvolver DM. (SBD, 2016)

\subsection{TRATAMENTO DA DM2}

Um dos pontos importantes no tratamento, controle da DM é a alimentação do paciente, tendo como uma das primeiras preocupações a redução de peso, já que a obesidade quase sempre está associada em pacientes com DM2. A dieta para o paciente portador de DM sugere um ingesta de $50 \%$ das calorias totais em forma de carboidratos, 15 a $20 \%$ de proteínas e 20 a $30 \%$ em gorduras. Ingesta de menos $2 \mathrm{~g}$ de sódio por dia. Paciente deve se alimentar com 5 a 6 refeições com horários e volumes controlados. (OLIVEIRA, 2014)

Figura 1 - Conduta no diabetes tipo 2

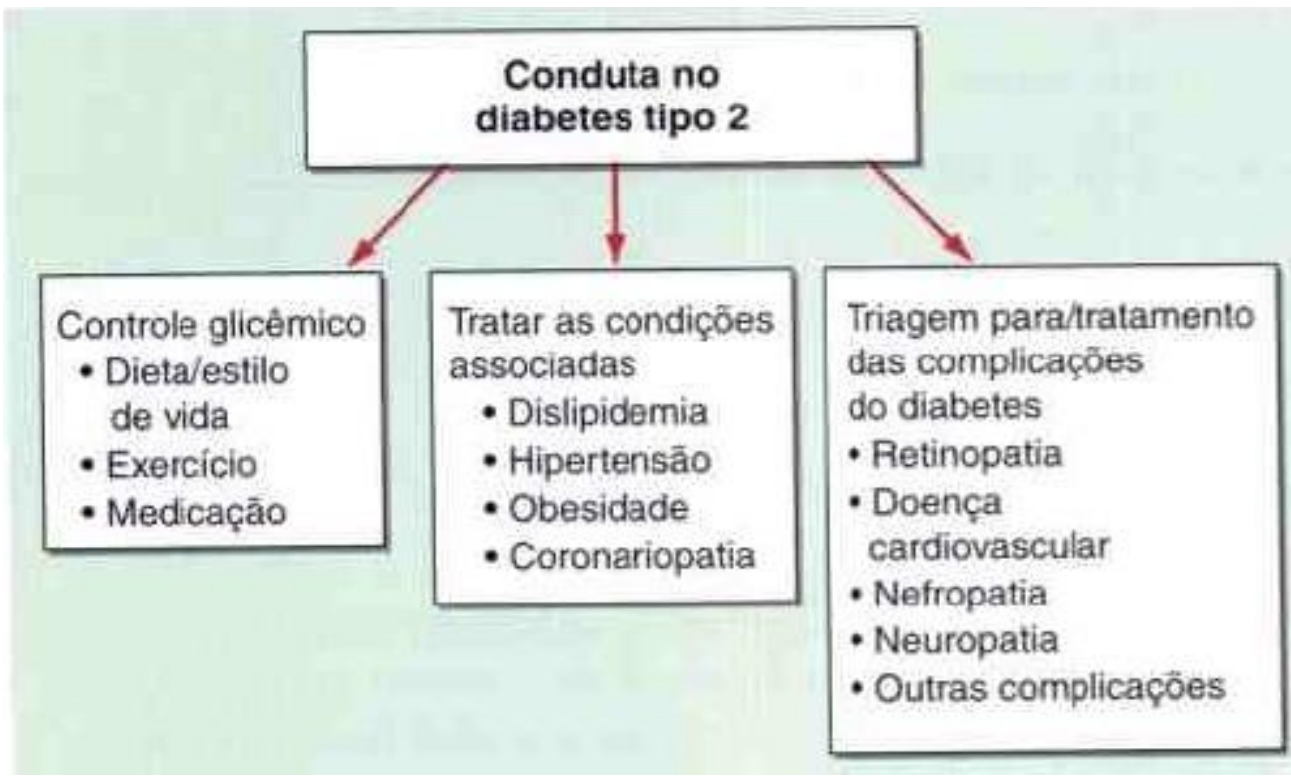

Fonte: Medicina Interna de Harrison (Volume 2), 2013, p. 2995

A figura 1 retrata as condutas a serem tomadas no tratamento da DM2. O controle glicêmico através de uma dieta ideal, mudança do estilo de vida sedentários com realização de exercícios e uso de medicamentos. Também devem ser acompanhadas e tratadas as doenças/condições que muitas vezes acompanham o paciente diabético como as: dislipidemias, HAS, obesidade e coronariopatias. Ficar vigilante perante as 
possíveis complicações que a DM pode trazer ao paciente entre elas as mais comuns são: retinopatia, doenças cardiovasculares, nefropatias e neuropatia. As complicações da DM serão melhores abordadas no capítulo dois desse trabalho. (POWERS, 2013)

"Os antidiabéticos orais são a primeira escolha de tratamento para pacientes com DM2 que não responderam bem as medidas não farmacológicas." (AURÉLIO, 2013, p.51)

Hipoglicemiantes orais: São úteis nos diabéticos tipo II e não gestacional que não respondem às medidas de dieta adequada, redução do excesso de peso e atividades físicas e cujo pâncreas ainda mantêm alguma produção de insulina. A decisão de iniciar hipoglicemiantes orais deve ser tomada com cuidado, após acompanhar o paciente por tempo suficiente para observar sua resposta e aderência ao tratamento convencional bem orientado, com alimentação adequada, redução do excesso de peso e atividade física. (OLIVEIRA, 2014, p. 570)

A escolha do medicamento (agente antidiabético) adequado deve considerar os valores das glicemias de jejum pós-prandial e da HbA1c, o peso e a idade do paciente, o estado geral do paciente, comorbidade presentes e as interações medicamentosas. (SBD, 2016) 
Figura 2 - Tratamento da DM2

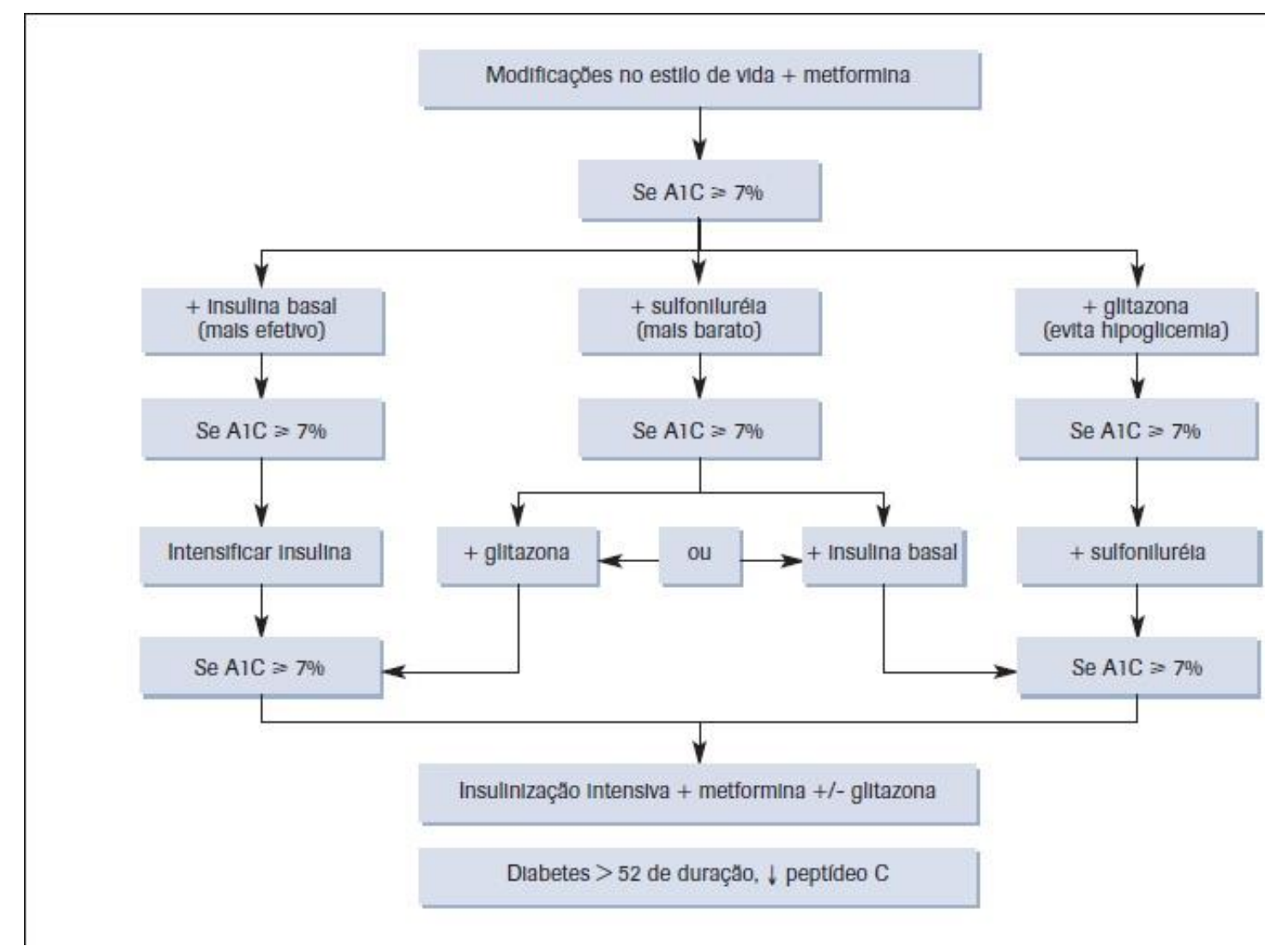

Fonte: Livro - Clínica Médica - USP (Volume 5), 2009, p. 326

O primeiro medicamento a ser inserido no tratamento do diabético tipo 2 e a metformina, que é de um grupo de medicamento conhecido como, biguanidas. Ela age diminuindo a produção hepática de glicose, desse modo ela reduz em média a HbA1c em 1,5 a 2\%. (SBD, 2016)

Se os níveis de A1c continuarem insatisfatórios, outra opção de tratamento são as sulfoniluréias, que agem aumentando a secreção de insulina. Elas atuam em um receptor especifico da membrana célula (SUR1), favorecendo o influxo de cálcio que estimula a secreção de insulina. As sulfoniluréias também são uma opção de tratamento mais barata. (SILVA et al., 2009)

As sulfoniluréias são "secretagogos" de insulina que agem através de receptores específicos de sulfoniluréias na superfície da célula beta. O ligante do receptor de drogas age para fechar os canais de potássio dependentes de adenosina trifosfato, 
resultando em despolarização celular, influxo de cálcio e translocação de grânulos secretórios de insulina para a superfície da célula. (SHERWIN; INZUCCHÍ, 2011, p. 2019)

As glitazona são sensibilizadores da ação da insulina, agem ativando os receptores nucleares PPAR-gama, ela também promove a adipogênese e aumenta o tecido adiposo subcutâneo, mais sensíveis a insulina. Reduzem a liberação de ácidos graxos livres, leptina, resistina e fator de necrose tumoral. Com essas reduções elas melhoram a sensibilidade à insulina no fígado e nos músculos, o que favorece a utilização da glicose e reduz a produção hepática de glicose. (SILVA et al., 2009)

Com perda cada vez maior da capacidade de secreção de insulina, grande maioria dos pacientes diabéticos no decorrer do tempo e evolução da doença, fara uso de insulinoterapia. Indicações para iniciar a insulinoterapia é persistência de glicemias acima de $230 \mathrm{mg} / \mathrm{dl} \mathrm{e} / \mathrm{ou} \mathrm{HbA} 1 \mathrm{c}$ acima de 8-9\%, mesmo com tratamento com outros medicamentos. (OLIVEIRA, 2014)

Tratamento com insulina no DM2, deve ser considerado como tratamento inicial nos pacientes magros que apresentam também redução ponderal acentuada, doença hepática, doença renal. (POWERS, 2013)

Há uma grande variedade de insulina, com diferentes tempos de ação, que envolve o tempo de início de suas ações, o seu pico de ação e seu tempo de duração. Com esses perfis de ação variados, e com os novos tipos de insulina, se tornou uma preocupação e que a insulinoterapia se torne muito complexa, e por isso ela seja ainda muito adiada. Com essa grande variedade de insulinas, o tratamento deve ser planejado de acordo com a resposta do paciente ao tratamento. (SBD, 2016)

\section{COMPLICAÇÕES DA DIABETES MELLITUS}

O paciente portador de DM2 poderá desenvolver com o decorrer do tempo várias complicações que surgem muitas vezes em consequência de um controle de glicêmico ruim, má adesão ao tratamento não medicamentoso e medicamentoso. 
Essas complicações podem implicar em sérios agravos a saúde, como, cegueira em decorrência da retinopatia diabética (RD), lesões/ulcerações, danos aos nervos devido a neuropatia diabética, danos aos rins ocasionada pela nefropatia diabética. Desse modo foi levantado informações sobre as principais complicações associadas a DM2.

\subsection{RETINOPATIA DIABÉTICA}

É a principal causa de cegueira entre pessoas com idade entre 24 a 74 anos e cerca de $60 \%$ dos pacientes doas pacientes com DM2 poderá desenvolver retinopatia diabética (RD) após 20 anos de doenças, ocasionando em perda da acuidade visual. (SBD, 2016).

O impacto econômico e social causado pelo DM, que já é considerado problema de saúde pública, deve-se em maior parte a essas complicações que encurtam a vida produtiva dos indivíduos, piorando sua qualidade de vida e a de seus familiares. (BOSCO, 2005, p. 2)

Em decorrência da perda parcial ou total da visão, pacientes com retinopatia diabética tem grande queda em sua qualidade de vida, tendo muitas vezes que se aposentar de suas atividades profissionais em decorrência dessa complicação e não podendo exercer suas atividades cotidianas como de costume antes do desenvolvimento dessa complicação.

A retinopatia diabética acontece devido a danos nos periquitos que são responsáveis pelo controle do fluxo sanguíneo, também havendo perda das células endoteliais capilares. Os primeiros sinais da retinopatia diabética é a formação de microaneurismas. (VIEIRA et al., 2009)

A RD pode ser classificada como, proliferativa e não proliferativa. $R D$ não proliferativa em média tem surgimento na primeira ou segunda década da doença, tem como características o desenvolvimento de microaneurismas vasculares retinianos, manchas algodonosas e exsudatos hemorrágicos. Sua fisiopatologia envolve o 
dano/desparecimento dos periquitos da retina, alteração na microvasculatura da retina, do fluxo sanguíneo e o aumento de permeabilidade vascular da retina. (POWERS, 2013)

RD proliferativa tem como principal característica a formação de neovasos (vasos novos) na vascularização da retina, esses vasos podem provocar hemorragias vítreas e o distinto descolamento de retina. Os vasos recém-formados podem aparecer próximos da mácula e/ou nervo óptico que acabam de rompendo-os com facilidade, formando assim, fibrose, hemorragia do vítreo. (VIERA et al., 2009; POWERS, 2013)

Quadro 2 - Classificação da RD

\begin{tabular}{|c|c|}
\hline $\begin{array}{l}\text { Gravidade da } \\
\text { retinopatia }\end{array}$ & $\begin{array}{l}\text { Achados à oftalmoscopia sob } \\
\text { dilataçäo pupilar }\end{array}$ \\
\hline Sem retinopatia aparente & Sem alteraçöes \\
\hline $\begin{array}{l}\text { Retinopatia diabética } \\
\text { não proliferativa leve }\end{array}$ & Microaneurismas apenas \\
\hline $\begin{array}{l}\text { Retinopatia diabética } \\
\text { não proliferativa } \\
\text { moderada }\end{array}$ & $\begin{array}{l}\text { Achados mais abundantes } \\
\text { que na retinopatia não proliferativa } \\
\text { leve, e menos abundantes que na } \\
\text { retinopatia não proliferativa grave }\end{array}$ \\
\hline $\begin{array}{l}\text { Retinopatia diabética } \\
\text { não proliferativa grave }\end{array}$ & $\begin{array}{l}\text { Presença de um dos seguintes } \\
\text { achados: mais de } 20 \text { hemorragias } \\
\text { retinianas em cada um dos quatro } \\
\text { quadrantes retinianos, } \\
\text { ensalsichamento venoso em dois } \\
\text { quadrantes ou microanormalidades } \\
\text { vasculares intrarretinianas em um } \\
\text { quadrante }\end{array}$ \\
\hline $\begin{array}{l}\text { Retinopatia diabética } \\
\text { proliferativa }\end{array}$ & $\begin{array}{l}\text { Presença de neovasos e/ou } \\
\text { hemorragia vitrea ou pré-retiniana }\end{array}$ \\
\hline
\end{tabular}

Fonte: SBD - Sociedade Brasileira de Diabetes, 2016

O quadro 3, faz a relação entre os achados na oftalmoscopia sob dilatação pupilar com a gravidade da retinopatia. $O$ exame pode variar desde o melhor resultado negativo que pode ser esperado que o de sem retinopatia aparente, quando não 
alterações no exame, até a forma mais grave da doença que seria a RD proliferativa que apresenta no exame a presença de neovasos, hemorragia vítrea ou de pré-retina.

\subsection{NEUROPATIA DIABÉTICA}

Cerca de $50 \%$ dos pacientes dos pacientes portadores de DM apresentam ou iram apresentar a neuropatia diabética (ND). O diagnóstico da neuropatia diabética só é dado quando excluídas todas as outras neuropatias possíveis, podendo ser detectado desde o início do diagnóstico de DM2. (SBD, 2016)

A ND pode ser dividida em neuropatia hiperglicêmica, neuropatia sensitivo-motora aguda, neuropatia sensitivo-motora crônica, neuropatias focal e multifocal. Sendo a mais comum entre elas a neuropatia sensitivo-motora crônica, tem como sintomas fisgadas, caibra, dormência, anestesia e fisgadas. Em sua forma grave podendo ter o surgimento de ulceras, artropatia de charcot e pode levar a amputação do membro como última medida terapêutica. (VIEIRA et al., 2009)

Sobre a fisiopatologia:

A lesão inicial e mais característica da polineuropatia crônica diabética é a desmielinização. Posteriormente, pode haver também degeneração axonal. $\mathrm{Na}$ polineuropatia aguda dolorosa, pode haver degeneração de fibras mielinizadas e nãomielinizadas. (VIEIRA et al., 2009, p. 339)

$\mathrm{Na}$ ND a glicose entra em níveis altos dentro dos nervos periféricos, sendo assim convertida em sorbitol pela enzima aldose redutase, esse acumulo de sorbitol no meio intracelular ocasiona uma diminuição do transporte ativo de vários metabolitos entre eles o mio-inositol. (DIAS; CARNEIRO, 2016)

A diminuição do mio-inositol altera os mecanismos de regulação intracelular, dessa forma, reduzindo a atividade da enzima sódio-potássio-ATPase que, por sua vez, reduz a atividade da bomba na/K, com o consequente acúmulo de sódio intracelular e alterações do potencial de repouso da membrana. Essas anormalidades diminuem a velocidade de condução neural e produzem as primeiras e reversíveis alterações 
estruturais nos nodos de Ranvier. De fato, foi demonstrado que inibidores da enzima aldose redutase normalizam ou melhoram várias funções alteradas do nervo periférico do diabético. (DIAS; CARNEIRO, 2016, p. 3)

No exame físico para auxilio no diagnóstico pode ser usado o monofilamento de Semmes-Weinstein (Figura 3), esse monofilamento exerce uma pressão de $10 \mathrm{~g}$ na região plantar. Podendo assim detectar o risco de ulceras, com sensibilidade no exame variando entre 86 e 100\%. (VIEIRA et al., 2009)

Figura 3 - Monofilamentos de Semmes-Weinstein
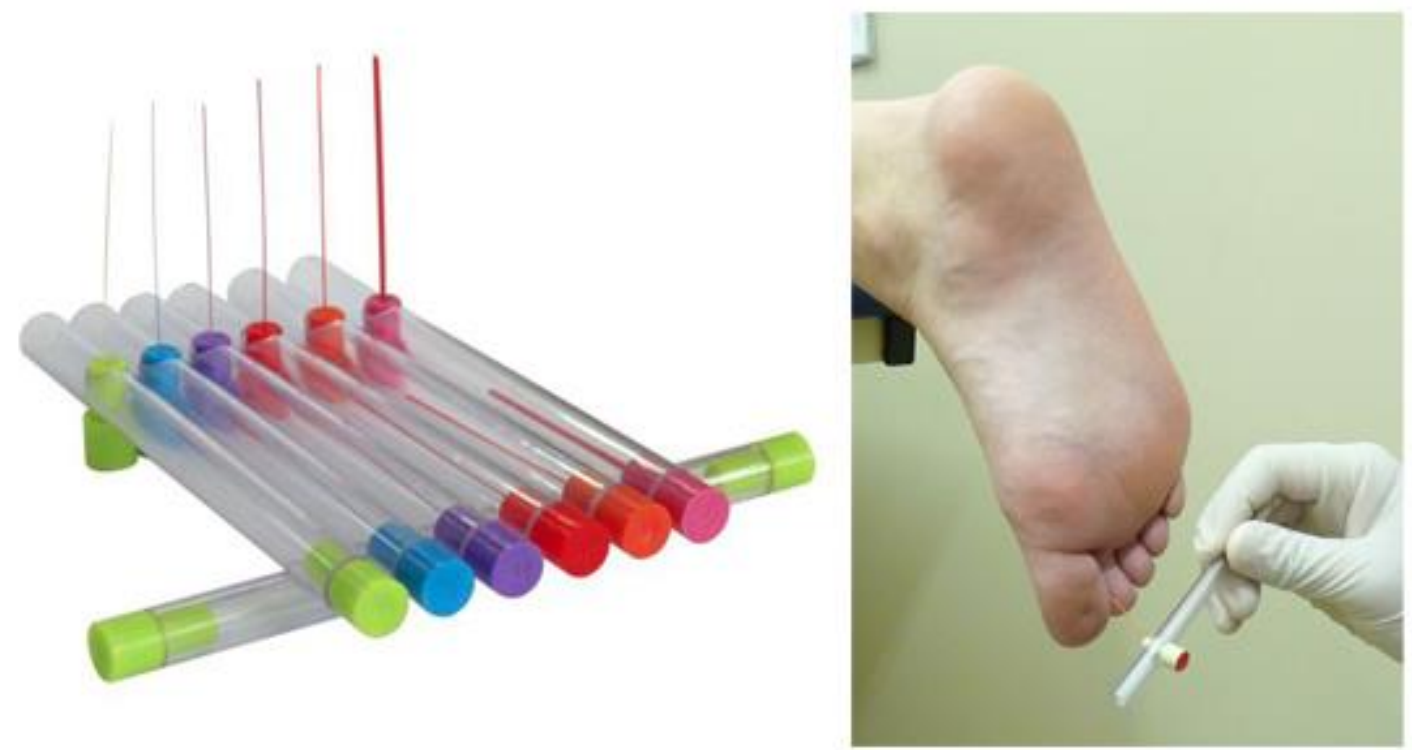

Fonte:http://biton.uspnet.usp.br/labimph/wpcontent/uploads/2014/05/monofilamentos. jpg

A sensibilidade vibratória também pode ser avaliada, por meio do diapasão de $128 \mathrm{~Hz}$ que nos casos da neuropatia o paciente pode apresenta ausência da presença de sensibilidade vibratória. Também pode ser utilizado o biotensiômetro, que pode variar sua vibração de 0 a 50 volts, seu exame e considerado positivo quando o paciente só sente vibração a partir de $25 \mathrm{~V}$, o que indica um risco para o desenvolvimento de úlceras. (VIEIRA et al., 2009) 
O tratamento da ND, tem como fator preventivo o controle metabólico, o que inibe o surgimento de lesões. Tratamento medicamentoso inclui:

"Ácido a-lipoico (age sobre a formação de espécies reativas de oxigênio), benfotiamina (previne o dano vascular no diabetes) e inibidores da aldose-redutase (reduz fluxo por meio da via do poliol)." (SBD, 2016, p. 134)

Quadro 3 - Tratamento ND sensitivo-motora

\begin{tabular}{|l|l|}
$\begin{array}{l}\text { Medicamentos } \\
\text { antidepressivos }\end{array}$ & Dose/dia \\
\hline Amitriptilina & 25 a $150 \mathrm{mg}$ \\
Duloxetina & 60 a $120 \mathrm{mg}$ \\
\hline Imipramina & 25 a $150 \mathrm{mg}$ \\
Nortriptilina & 10 a $150 \mathrm{mg}$ \\
Venlafaxina & 150 a $225 \mathrm{mg}$ \\
\hline $\begin{array}{l}\text { Medicamentos } \\
\text { anticonvulsivantes }\end{array}$ & Dose/dia \\
\hline Gabapentina & 900 a $1.800 \mathrm{mg}$ \\
Pregabalina & 150 a $600 \mathrm{mg}$ \\
\hline
\end{tabular}

Fonte: SBD - Sociedade Brasileira de Diabetes, 2016

Uma das indicações no quadro 3 e a amitriptilina alivia a dor por meio da inibição da recaptação da norepinefrina e de serotonina nas sinapses das vias descendentes da dor a iniciando do sistema nervoso central e do receptor da N-metil-D-aspatato, que funciona como mediador da alodinia e hiperalgesia. (VIEIRA et al., 2009)

\subsection{NEFROPATIA DIABÉTICA}

A nefropatia diabética (NFD) é baseada na excreção de albumina taxa de filtração glomerular. Em portadores de DM2 tem incidência de 5 a $20 \%$ e é a principal causa de doença renal crônica responsável por paciente necessitarem de dialise. Hoje em dia a NFD é definida pela proteinúria detectável persistente, geralmente associada a elevação da pressão arterial. Ficou comprovado que o desenvolvimento NFD aumenta 
o risco de eventos cardiovasculares e o índice de mortalidade nos pacientes portadores de DM2. (SBD, 2016)

Todo esse efeito negativo, em decorrência do aumento plasmático da glicose no sangue, que aumenta a taxa de filtração glomerular (TFG), que pode vim acompanhada de uma hipertensão glomerular que resulta em lesão endotelial, com a diminuição de óxido nítrico e aumento de angiotensina 2, isso pode gerar uma glomeruloesclerose, lesão histológica, microaneurismas, nessa fase é encontrada microalbuminúria. (VIEIRA et al., 2009)

Segundo Harris (2009) a nefropatia diabética pode ser divididas em quatro estagios sendo eles:

Figura 4 - Estágios da NFD

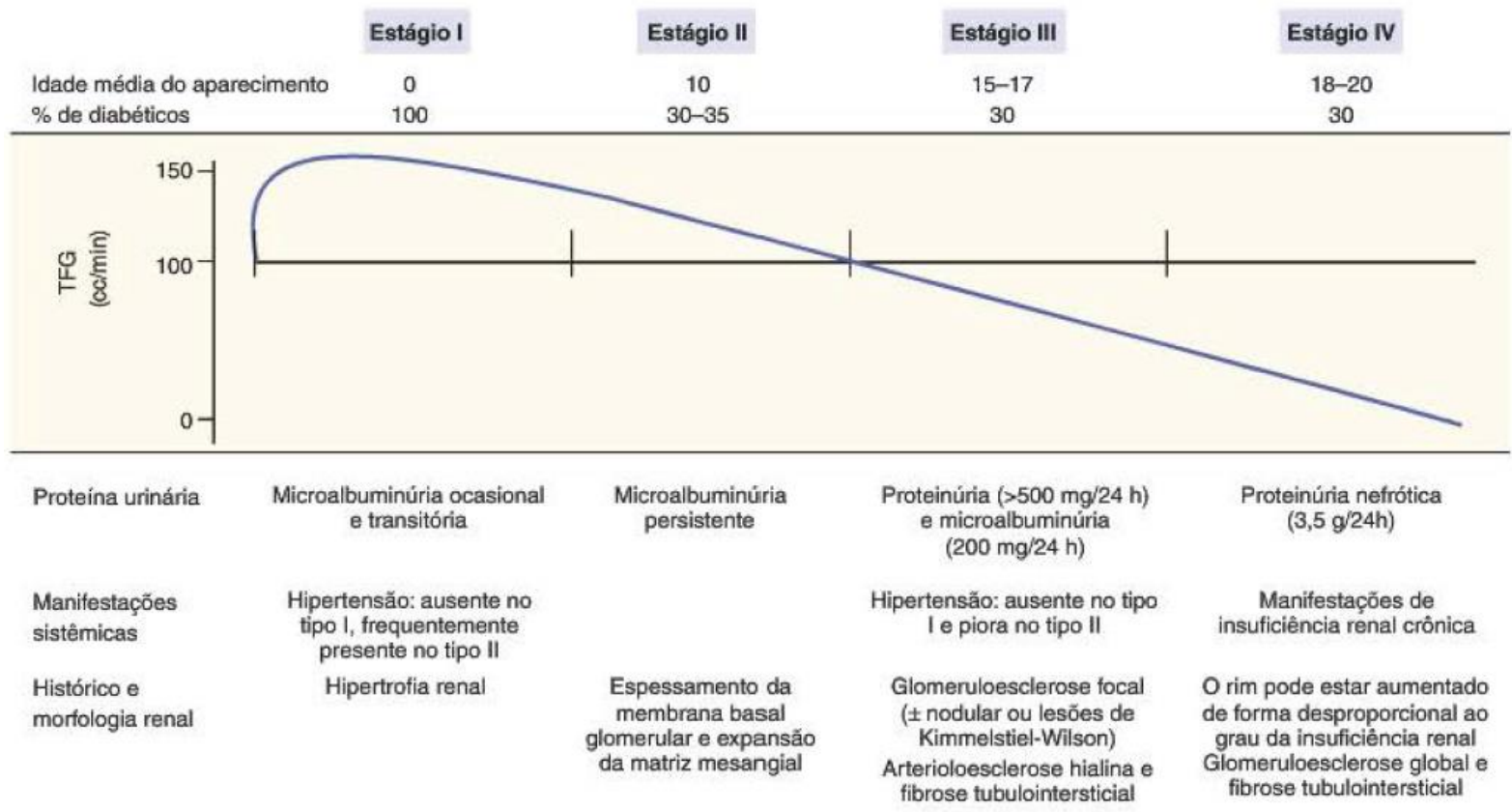

Fonte: Cecil Medicina, 2009, p.1025.

Estágio 1: os rins sofrem uma hipertrofia de glomérulos e túbulos e a um aumento do fluxo sanguíneo renal e da taxa de filtração glomerular. Não há uma macroalbuminúria detectável nesse estágio. Pode ser observada uma hipertensão nos pacientes portadores de DM2 como informado na figura 4. (HARRIS, 2009) 
Estagio 2: Tem como característica a presença de microalbuminuria. A evolução para o estágio 2 e silenciosa, podendo demorar 10 anos para atingir esse estágio. A uma alteração na histologia normal, que tem característica o espessamento das membranas e o início da expansão da matriz mesangial. $O$ desenvolvimento de microalbuninúria já se torna aumentada, provocando agressões microvasculares. A hipertensão por si só pode causar microalbuminúria. Como é ilustrado na figura 4 (HARRIS, 2009)

Estágio 3: Pacientes que tem uma microalbuminúria, acabam evoluindo para uma nefropatia franca, que é o estágio 3 em um período de 5 a 7 anos. A uma proteinúria explícita, que é uma concentração superior a 500 mg de proteína total em 24 horas e uma microalbuminúria, uma concentração superior a 200 mg em 24 horas, como demostrado na figura 4. Nessa fase os pacientes com DM2 que também sejam hipertensos, encontram uma dificuldade no controle da pressão arterial. Na NFD explícita, ocorre a fibrose tubulointersticial progressiva e a TFG começa a entrar em declínio, mas o nível de creatinina sérica pode permanecer normais. (HARRIS, 2009)

Estágio 4: é a NFD avançada, com a função renal bastante debilitada, o paciente apresenta uma proteinúria com valores alarmantes, extremamente tóxicos, valores superiores a 3,5 g em 24 horas. Em pacientes portadores de DM2 tem maior incidência de microalbuminúria e hipertensão arterial. (HARRIS, 2009)

\section{ASSISTÊNCIA DE ENFERMAGEM AO PORTADOR DE DIABETES TIPO DOIS}

O enfermeiro como membro da equipe multidisciplinar, deve buscar meios de fazer a promoção da saúde. Um dos principais pontos a serem trabalhados é a prevenção, usando de métodos para evitar que pacientes de risco (história familiar de DM2, má alimentação e sedentários) desenvolvam DM2.

Muitos pacientes tem um conhecimento deficiente em se tratando de DM2, muitos tem tempo a patologia há anos, não sabem, definir o que é a doença ou valores normais de glicose. No estudo feito por Pace (2006), somente 8,6 dos pacientes no estudo 
acertaram os valores de normalidade de glicose, de $70-110 \mathrm{mg} / \mathrm{dl}$. Isso pode mostrar uma enorme dificuldade, devido ao mal entendimento sobre a doença, o que pode levar o paciente não entender a gravidade da patologia e as consequências que ela pode causa caso não seja controlada.

\subsection{ATUAÇÃO DO ENFERMEIRO NA PREVENÇÃO}

A maior meio do enfermeiro agir na prevenção da DM2 e através da educação. O enfermeiro através da consulta de enfermagem poderá filtrar/detectar fatores de risco, entre eles a hereditariedade, obesidade, sedentarismo entre outros que são mostrados no quadro 5 , que podem levar a o desenvolvimento da DM2.

Quadro 4 - Critérios de rastreamento da DM

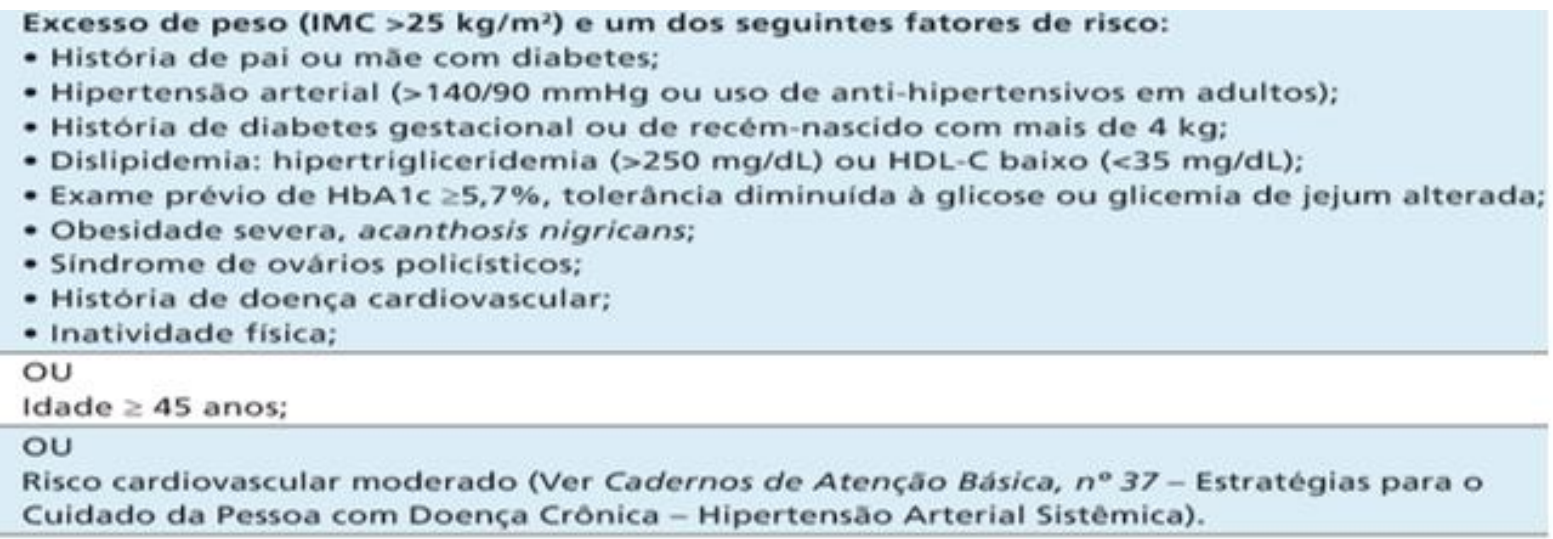

Fonte: ADA - Sociedade Americana de Diabetes, 2017

\subsection{ASSISTÊNCIA DE ENFERMAGEM AO DIAGNÓSTICO E ACOMPANHAMENTO}

Os pacientes com fatores de risco devem ser encaminhados para uma consulta na qual será feito o rastreamento da DM e será realizado o exame de glicemia. Paciente com resultado do teste de glicose alterar, para a intolerância à glicose poderão realizar o acompanhamento anualmente para. (MINISTÉRIO DA SAÚDE, 2013)

Recomenda-se que a consulta de rastreamento para a população-alvo definida pelo serviço de Saúde seja realizada pelo enfermeiro da UBS, encaminhando para o 
médico em um segundo momento, a fim de confirmar o diagnóstico dos casos suspeitos. (MINISTÉRIO DA SAÚDE, 2013, p. 28)

Essa consulta tem objetivo de rastreamento, buscando conhecer a história pregressa da pessoa, fazer anamnese e realizar o exame físico que inclui a coleta de dados antropométricos, aferição de pressão arterial, cálculo de IMC, buscar pelos fatores, avaliar exames laboratoriais, interpretando se seu resultado pode ter alguma repercussão nas condutas terapêuticas da DM2.

O enfermeiro em sua consulta rotineira, vendo que o paciente tem sintomas clássicos da DM, deverá pedir para o profissional médico a solicitação de exames para o paciente que apresenta esses sinais e sintomas que indica o diagnóstico de DM. (MINISTÉRIO DA SAÚDE, 2013) 
Figura 5 - Rastreamento paciente diabético

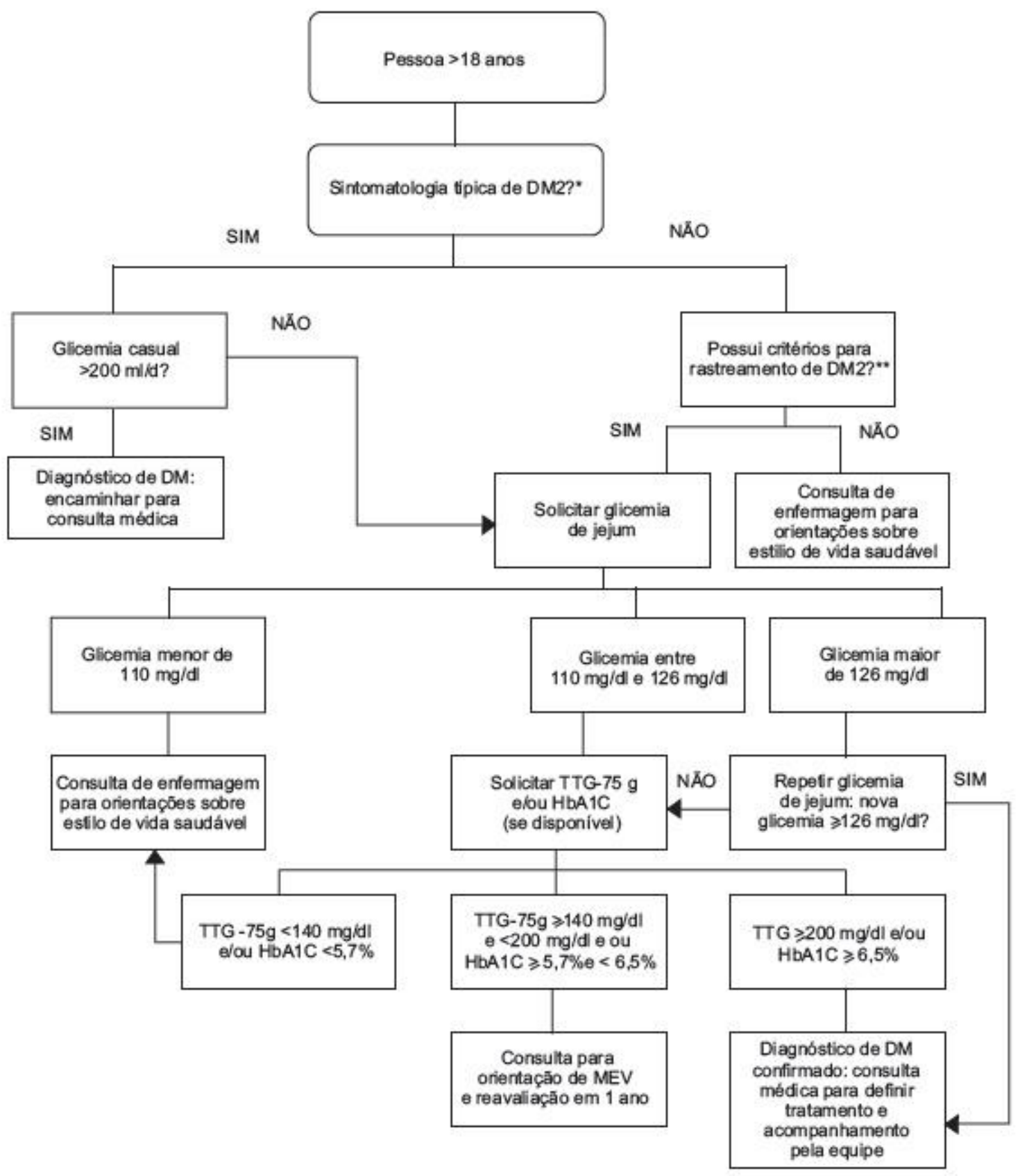

Fonte: Ministério da Saúde, Caderno de Atenção básica no 36, 2013

É mostrado na figura 5, os passos que devem ser seguidos para o possível diagnóstico de DM. Paciente com idade superior a 18 anos com sintomatologia, se esse mesmo paciente tiver uma glicemia casual superior a $200 \mathrm{mg} / \mathrm{dll}$, o paciente deve ser encaminhado para a consulta médica para se diagnosticado com DM. Se o paciente não apresentar glicemia casual superior a $200 \mathrm{mg} / \mathrm{dl}$, deve ser feito uma glicemia em

$\mathrm{RC}: 18971$

Disponível em: https://www.nucleodoconhecimento.com.br/saude/enfermagem-ao-paciente 
jejum, se resultado entre $110-126 \mathrm{mg} / \mathrm{dl}$, deve ser solicitado o teste de tolerância a glicose, se maior de $126 \mathrm{mg} / \mathrm{dl}$ deve ser repetido a glicemia em jejum, se novamente de outro resultado a superior a $126 \mathrm{mg} / \mathrm{dl}$ ou teste de tolerância a glicose superior a $200 \mathrm{mg} / \mathrm{dl}$ o paciente dever ser encaminhado para consulta médica para se diagnosticado.

\subsection{CONSULTA DE ENFERMAGEM}

Em vista as mudanças que são necessárias na vida do paciente portador de DM, o enfermeiro tem que o orientas sobre medidas para o controle efetivo da DM, medidas que envolve a mudança no estilo de vida do paciente. Através da consulta de enfermagem e colhido dados relevantes para as condutas do enfermeiro, que irá identificar possíveis problemas que o paciente poderá enfrentar no seu dia-a-dia, buscando soluciona-los de modo a conseguir os efeitos terapêutico esperados.

A assistência de enfermagem para a pessoa com DM precisa estar voltada para um processo de educação em saúde que auxilie o indivíduo a conviver melhor com a sua condição crônica, reforce sua percepção de riscos à saúde e desenvolva habilidades para superar os problemas, mantendo a maior autonomia possível e tornando-se corresponsável pelo seu cuidado. As ações devem auxiliar a pessoa a conhecer o seu problema de saúde e os fatores de risco correlacionados, identificar vulnerabilidades, prevenir complicações e conquistar um bom controle metabólico que, em geral, depende de alimentação regular e de exercícios físicos. (MINISTÉRIO DA SAÚDE, 2013, p. 35)

$\mathrm{Na}$ consulta de enfermagem será colhido o histórico do paciente, que inclui: Identificação do paciente, seus antecedentes pessoais e familiares, suas queixas, medicamentos em utilização, seus hábitos diários e a identificação de fatores de risco. (MINISTÉRIO DA SAÚDE, 2013)

No exame físico deverá ser colhido e avaliado a altura peso, circunferência abdominal, IMC, pressão arterial, frequência cardíaca e respiratória, alterações visuais, avaliação de membro (verificando presença de ulceras, mobilidade, temperatura, sensibilidade 
e edemas), avaliação da pele (verificar coloração, integridade, turgor). (MINISTÉRIO DA SAÚDE, 2013)

Através das informações colhidas o enfermeiro poderá fazer diagnósticos de enfermagem que poderá guiar suas decisões. Os diagnósticos de enfermagem que podem ser muitas vezes encontrados em paciente são:

Segundo Deitos (2015), autonegligência, conhecimento deficiente, estilo de vida sedentário, fadiga, integridade da pele prejudicada, nutrição desequilibrada: mais do que as necessidades corporais, risco de disfunção neurovascular periférica, risco de glicemia instável, risco de lesão, risco de perfusão renal ineficaz. Alguns desses diagnósticos vão estar mais presentes em caso de complicações crônicas, exemplo, integridade da pele prejudicada, bastante presente em pacientes com neuropatia diabética, na qual pode desenvolver úlceras.

\subsection{CONDUTAS DE ENFERMAGEM - PRÉ DIABÉTICO}

Está relacionado a neuropatia diabética, que causa a perda de sensibilidade muitas vezes de membros inferiores (MMII) no qual surge ulcerações de variados diâmetros e profundidades. Em suas apresentações graves podem gerar a amputação dos quirodáctilos afetados, de todo o pé afetado ou até mesmo amputação infra-femoral. (SBD, 2016; MINISTÉRIO DA SAÚDE, 2013)

Embora a deficiência primária da diabetes seja de natureza metabólica, a causa sobreposta de morbilidade do pé diabético é biomecânica. Na maior parte dos casos a causa da lesão não é a infecção ou isquemia, mas sim a agressão mecânica que a insensibilidade neuropática permite. (DUARTE; GONÇALVES, 2011, p. 69-70)

Desse modo o enfermeiro deve buscar no momento da consulta fatores de risco de podem contribui para o desenvolvimento do pé diabético, como: tabagismo, NFD em tratamento de diálise, doença vascular periférica, deformidade nos pés, mau controle glicêmico, alguma amputação prévia. (MINISTÉRIO DA SAÚDE, 2013) 
O paciente deve ser questionado sobre a presença de sintomas comuns para essa complicação como sensação de choque, queimação, dor e dormência. Lembrando que o controle rigoroso da glicemia pode evitar o desenvolvimento dessa complicação. (ADA, 2017; SBD, 2016; MINISTÉRIO DA SAÚDE, 2013)

O enfermeiro atua diretamente no tratamento da lesão ocasionada pelas ulcerações através da avaliação da ferida, realização de desbridamento (mecânico, autolítico e enzimático) e utilizando coberturas adequadas para o curativo de acordo com sua avaliação. (HIROTA; HADDAD; GUARIENTE, 2008)

Deverá ser escolhida uma cobertura ideal para o curativo, entre essas coberturas podemos encontrar, a placa de hidrocolóide que é indicada para feridas com pouco a moderado exsudato; hidrogel que é indicado feridas com necrose; alginato de cálcio indicado para feridas com pouco a moderado exsudato; carvão ativado indicado para feridas infectadas, com odor fétido e que tenha pouco exsudato; espuma de poliuretano com prata indicado para feridas com moderado a alta produção de exsudato, infectadas ou não. (CARMO, 2007)

\subsection{CONDUTAS NAS DEMAIS COMPLICAÇÕES}

Mediante as outras complicações o enfermeiro age esclarecendo o quadro clinico ao paciente junto com a equipe multidisciplinar, determinando os cuidados que ele deve ter para controlar da maneira mais efetiva patologia.

O enfermeiro deve:

- Orientar o paciente o modo e a importância da aferição diária dos níveis de glicose;

- Orientar e informar sobre as complicações que podem surgir casos o tratamento não seja realizado de maneira eficaz;

- Realizar exame físico, buscando sinais que indicam complicações; 
- Piora na acuidade visual, devem ser investigadas, pois pode ser uma manifestação de retinopatia diabética. Paciente deverá ser encaminhado para consulta médica;

- Deverá estar atendo a sinais de anestesia em membros, o que é indicativo de uma neuropatia em curso;

- O enfermeiro pode prescrever medicamentos que estejam previstos em protocolos institucionais. (AURÉLIO, 2013; BETÕNICO, 2016; FAEDA; LEON, 2006)

\section{CONSIDERAÇÕES FINAIS}

O capítulo um teve seu objetivo alcançado, que foi de esclarecer os principais aspectos da diabetes mellitus, demostrando detalhes sobre sua epidemiologia, seu processo de fisiopatologia, modo que é realizado o diagnóstico de diabetes mellitus e abordar o tratamento da diabetes mellitus e os diversos fármacos que podem estar envolvidos nesse tratamento.

Foi abordado no capítulo dois as complicações mais comumente encontradas nos pacientes portadores da diabete mellitus tipo dois, sendo elas: retinopatia diabética, neuropatia diabética, nefropatia diabética. Foram relata as características de cada complicação, incluindo sua definição, classificações, desse modo alcançando o objetivo proposto.

No terceiro capítulo foi relatado as condutas de enfermagem frente ao paciente diabético, retratando a atuação do enfermeiro na prevenção da diabetes mellitus; cuidados em uma das complicações que lida com mais autonomia que é a neuropatia diabética ou pé diabético e suas condutas relação as demais complicações crônicas comuns na diabetes mellitus tipo dois.

\section{REFERÊNCIAS}

AURÉLIO, Hêider Pinto. Ministério da Saúde (Ed.). Caderno de Atenção Básica: ESTRATÉGIAS PARA O CUIDADO DA PESSOA COM DOENÇA CRÔNICA 
- DIABETES MELLITUS. Brasília: Ministério da Saúde, 2013. 162 p. (36). Disponível em: $\quad$ http://189.28.128.100/dab/docs/portaldab/publicacoes/caderno_36.pdf>. Acesso em: 30 ago. 2017.

ADA - American Diabetes Association. STANDARDS OFMEDICAL CARE IN DIABETES. Ed. Diabetes Care, 2017. Disponível em: $<$ http://professional.diabetes.org/sites/professional.diabetes.org/files/media/dc_40_s1 final.pdf $>$. Acesso em: 24/04/2017.

BETÕNICO, Carolina de Castro Rocha et al. Complicações Crônicas do Diabete Melito. In: MARTINS, Mílton de Arruda et al (Ed.). Clínica Médica. 2. ed. São Paulo: Manole, 2016. Cap. 28. p. 352-365.

BOSCO, Adriana et al. Retinopatia diabética. Arq. bras. endocrinol. Metab, v. 49, n. 2, p. 217-227, 2005.

CARMO, Sara da Silva et al. Atualidades na assistência de enfermagem a portadores de úlcera venosa. 2007. Disponível em: http://dms.ufpel.edu.br/ares/bitstream/handle/123456789/185/7208-26991-1PB.pdf?sequence=1 >. Acesso em: 07/10/2017

DEITOS, Dieimi Lopes. NANDA Internacional - Diagnósticos de Enfermagem da Nanda: Definições e Classificação - Nanda International. 10. ed. Brasil: Artmed, 2015.

DIAS, Rafael José Soares; CARNEIRO, Armando Pereira. Neuropatia diabética: fisiopatologia, clínica e eletroneuromiografia. Acta Fisiátrica, v. 7, n. 1, p. 35-44, 2016. Disponível em: http://www.revistas.usp.br/actafisiatrica/article/viewFile/102254/100636. Acesso em: 06/08/2017

DUARTE, Nádia; GONÇALVES, Ana. Pé diabético. Angiol Cir Vasc, Lisboa, v. 7, n. 2, p. 65-79, jun. 2011. Disponível em <http://www.scielo.mec.pt/scielo.php?script=sci_arttext\&pid=S1646706X2011000200002\&lng=pt\&nrm=iso>. Acessos em 08 out. 2017. 
FAEDA, Alessandra; LEON, Cassandra Genoveva Rosales Martins Ponce de. Assistência de enfermagem a um paciente portador de Diabetes Mellitus. Revista Brasileira de Enfermagem, Brasília, v. 59, n. 6, dez. 2006. Disponível em: $<$ http://www.scielo.br/scielo.php?script=sci_arttext\&pid=S003471672006000600019\&lng=pt\&nrm=iso >. Acesso em: 06/05/2017.

FERREIRA, Sandra Roberta Gouvea; PITITTO, Bianca de Almeida. E-Book 2.0 Diabetes na Prática Clínica - Capítulo 1 - Aspectos epidemiológicos do Diabetes Mellitus e seu impacto no indivíduo e na sociedade. Ed. 2014.SBD - Sociedade Brasileira $\mathrm{De}$ Diabetes. em: $<$ http://www.diabetes.org.br/ebook/component/k2/item/73-capitulo-1-aspectosepidemiologicos-do-diabetes-mellitus-e-seu-impacto-no-individuo-e-na-sociedade>. Acesso em: 04/05/2017.

HARRIS, Raymond C.. DIABETES MELLITUS E RIM. In: GOLDMAN, Lee; AUSIELLO, Dennis (Ed.). Cecil Medicina. 23. ed. Rio de Janeiro: Elsevier, 2009. Cap. 125. p. 1023-1028.

HIROTA, Cristina Miyuki Okumoto; HADDAD, Maria do Carmo Lourenço; GUARIENTE, Maria Helena Dantas de Menezes . Pé diabético: o papel do enfermeiro no contexto das inovações terapêuticas-DOI: 10.4025/cienccuidsaude. v7i1. 4955. Ciência, Cuidado e Saúde, v. 7, n. 1, p. 114-120, 2008.

INTERNATIONAL DIABETES FEDERATION. IDF Diabetes Atlas [Internet]. 6a ed. Brussels: International Diabetes Federation, 2015.Disponível em: <http://www.idf.org/diabetesatlas>. Acesso em: 25/04/2017

MINISTÉRIO DA SAÚDE. CADERNOS DE ATENÇÃO BÁSICA: ESTRATÉGIAS PARA O CUIDADO DA PESSOA COM DOENÇA CRÔNICA - DIABETES MELLITUS. Brasília: Ministério da Saúde, 2013. Disponível em: $<$ http://bvsms.saude.gov.br/bvs/publicacoes/estrategias_cuidado_pessoa_diabetes_ mellitus_cab36.pdf>. Acesso em: 07 set. 2017. 
NAM HAN CHO. Idf - Federação Internacional de Diabetes (Org.). Atlas de la DIABETES de la FID. 7. ed. Bélgica: International Diabetes Federation, 2015. 144 p. Disponível em: <http://www.diabetesatlas.org/>. Acesso em: 29 ago. 2017.

OLIVEIRA, Gésica Kelly da Silva; OLIVEIRA, Emanuela Rozeno de. Assistência De Enfermagem Ao Portador De Diabetes Mellitus: Um Enfoque Na Atenção Primária Em Saúde. Veredas Favip, Caruaru, v. 2, n. 3, p.40-48, jul. 2010. Disponível em: <http://veredas.favip.edu.br/ojs/index.php/veredas1/article/view/96>. Acesso em: 24/04/2017.

OLIVEIRA, Reynaldo Gomes de. Black Book: Clínica Médica. 2. ed. Belo Horizonte: Blackbook Editora Ltda., 2014. 789 p.

OMS - ORGANIZAÇÃO MUNDIAL DA SAÚDE. INFORME MUNDIAL SOBRE LA DIABETES. Genebra: Oms, 2016. 4 p. Disponível em: <http://apps.who.int/iris/bitstream/10665/204877/1/WHO_NMH_NVI_16.3_spa.pdf?u $\mathrm{a}=1>$. Acesso em: 05 set. 2017.

PACE, Ana Emilia et al. 0 conhecimento sobre diabetes mellitus no processo de autocuidado. Revista Latino-Americana de Enfermagem, Ribeirão Preto, v. 14, n. 5, p. 728-734, oct. 2006. ISSN 1518-8345. Disponível em: <http://www.revistas.usp.br/rlae/article/view/2355/2535>. Acesso em: 15 feb. 2017. doi:http://dx.doi.org/10.1590/S0104-11692006000500014.

POWERS, Alvin C.. Diabetes Melito. In: LONGO, D. L. et al. Medicina Interna de Harrison. 18. ed. New York: Amcg Editora Ltda, 2013. Cap. 344. p. 2968-3003.

SBD - SOCIEDADE BRASILEIRA DE DIABETES. Diretrizes da Sociedade Brasileira de Diabetes. Ed. 2015-2016a. Disponível em: $<$ <ttp://www.diabetes.org.br/profissionais/images/pdf/DIRETRIZES-SBD-20152016.pdf>. Acesso em: 24/04/2017. 
. Atlas do Diabetes 2015 - Atualização. Ed. 7. 2015b. Disponível em: < http://www.diabetes.org.br/profissionais/images/pdf/atlas-idf-2015.pdf>. Acesso em: 04/05/2017.

. Cuidados de Enfermagem em Diabetes Mellitus. In: GROSSI, Sonia aurora Alves; PASCALI, Paula Maria. São Paulo. 2009c.

SILVA, Maria Elizabeth Rossi da et al (Ed.). Diabetes Mellitus. In: MARTINS, Mílton de Arruda et al (Ed.). Clínica Médica. São Paulo: Manole, 2009. Cap. 29. p. 311-329. Volume 5.

SHERWIN, Robert S; INZUCCHÍ, Silvio E. Diabetes Mellitus tipo 2. In: LNZUCCHÍ, Silvio E. (Org.). Cecil: Medicina Interna. 23. ed. Rio de Janeiro: Elsevier, 2011. Cap. 248, p. 2014.

VIEIRA, Suzana Maria de Souza et al. Complicações do Diabetes. In: MARTINS, Mílton de Arruda et al (Ed.). Clínica Médica. Barueri: Manole, 2009. Cap. 30. p. 330342. Volume 5. 